\title{
DINÂMICA HIDROSSEDIMENTOLÓGICA NA BACIA DO RIO GUABIROBA, GUARAPUAVA, PARANÁ
}

\author{
HYDROSEDIMENTOLOGICAL DYNAMICS IN THE GUABIROBA RIVER CATCHMENT, \\ GUARAPUAVA, PARANÁ
}

\author{
Fernanda BORTOLATTO ${ }^{1}$, Leandro Redin VESTENA ${ }^{2}$ \\ ${ }^{1}$ Programa de Pós-Graduação em Geografia, Universidade Estadual do Centro-Oeste, UNICENTRO. Alameda Élio Antônio Dalla \\ Vecchia, 838, Guarapuava - PR. E-mail: fernandabortolatto@gmail.com \\ ${ }^{2}$ Universidade Estadual do Centro-Oeste. Laboratório de Hidrologia, Campus Cedeteg. Alameda Élio Antônio Dalla Vecchia, 838, \\ Guarapuava - PR. E-mail: lvestena@unicentro.br
}

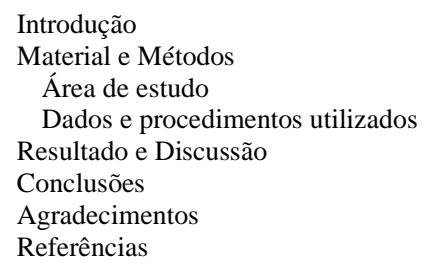

RESUMO - A histerese entre vazão e concentração de sedimentos manifesta a dinâmica hidrossedimentológica na bacia hidrográfica, em função da produção e transporte de sedimentos, determinada principalmente pelo uso e manejo do solo, precipitação e concectividade hidrogeomorfológica. O presente trabalho apresenta os resultados de estudo sobre a dinâmica hidrossedimentológica em uma bacia hidrográfica rural, no Sul do Brasil, a partir da classificação e análise das curvas de histerese. A pesquisa avaliou vinte eventos hidrológicos monitorados na bacia do rio Guabiroba, em Guarapuava, Paraná. A classificação das curvas de histerese deu-se em função da dinâmica de comportamento da vazão e da concentração de sedimento em suspensão ocorrida nos eventos pluviométricos, em um dos tipos de modelos de histerese descritos na literatura. Os resultados obtidos demostraram ocorrer na bacia cinco tipo de modelo de histerese, principalmente, as do tipo clockwise loop e figure eight. A relação entre a vazão e a concentração de sedimento em suspensão total, na ascensão e recessão do hidrograma apresentou coeficiente de correlação positiva na maioria dos eventos. As características apresentadas nas diversas curvas de histerese na bacia hidrográfica são resultado da disponibilidade de sedimentos finos ao transporte fluvial, provenientes da erosão das vertentes, das margens fluviais, e de estradas rurais.

Palavras-chave: Histerese. Sedimento em suspensão. Erosão. Transporte de sedimento.

\begin{abstract}
Hysteresis between flow and sediment concentration manifests the hydrosedimentological dynamics in the catchment, depending on the production and transport of sediments, mainly determined by the use and management of the soil, precipitation and hydrogeomorphological connectivity. This paper presents the results of a study of hydrosedimentological dynamics in a rural river basin in southern Brazil, based on classification and analysis of hysteresis curves. The study evaluated twenty hydrological events monitored in the Guabiroba river basin, in Guarapuava, Paraná. The hysteresis curves were classified according to flow behavior dynamics and suspended sediment concentration that occurred during rainfall events, according to types of hysteresis models described in the literature. The results obtained demonstrated that five types of hysteresis models occur in the basin, mainly clockwise loop and figure eight. The relationship between flow and concentration of totally suspended sediment, as per hydrograph rise and fall, showed a positive correlation coefficient in most events. The characteristics presented in the different hysteresis curves in the river basin are the result of the availability of fine sediments for river transport, resulting from the erosion of slopes, river banks, and rural roads.
\end{abstract}

Keywords: Hysteresis. Suspended sediment. Erosion. Sediment transport.

\section{INTRODUÇÃO}

O diagnóstico do comportamento hidrossedimentológico de uma bacia hidrográfica, pode ser determinado a partir da análise da relação entre concentração de sedimento suspenso (Css) e vazão (Q).

$\mathrm{O}$ resultado dessa relação resulta em uma figura chamada de histerese. A histerese explicita a evolução de um fenômeno físico em relação ao outro.

No caso, a variação da vazão impacta na concentração de sedimento suspenso.

De modo geral, nas menores vazões têm-se o transporte de sedimentos mais finos e nas maiores os sedimentos de maior granulometria. Porém, durante os eventos de chuva, a relação existente entre a vazão e a concentração de sedimentos, normalmente, não é homogênea, apresentando um comportamento complexo (Lopes et al., 2007).

A histerese reflete a complexidade dos picos de Q e Css que muitas vezes não coincidem, assim como as taxas de ascensão e recessão de cada uma das variáveis, dada por um atraso na evolução de um dos fenômenos físicos em 
relação ao outro, uma falta de sincronia na dinâmica (Klar, 1984; Klein, 1984; Williams, 1989; Jansson, 2002; Stott, 2006; Lefrancois et al., 2007, Vestena, 2009), condicionando diferentes comportamentos hidrossedimentológicos e histereses.

O entendimento da dinâmica hidrossedimentológica em bacia hidrográfica rural é de suma importância à medida que subsidia ações para conservação do solo e da água.

Os sedimentos transportados decorrem de processos erosivos atuantes na bacia hidrográfica que deterioram a qualidade do solo, e a presença de sedimentos finos nos cursos fluviais, produzem turbidez e atuam como portadores de poluentes (herbicidas, inseticidas e fungicidas), deteriorando a qualidade da água.

A análise qualitativa de curva de histerese realizada por Williams (1989), identificou e classificou cinco tipos: valor simples (singlevalued); sentido horário (clockwise); sentido anti-horário (counterclockwise); linha simples comum (single line plus); e figura oito (figure eight).

Cada um dos tipos de curva de histerese é resultado de um dinâmica hidrossedimentológica específica (Williams, 1989; Jansson, 2002; Lenzi \& Marchi, 2000; Seeger et al., 2004; Baca, 2008; Vestena, 2009):

(I) Single-valued - curva obtida quando o pico de Css e vazão ocorrem simultaneamente.

Este tipo de histerese corresponde à relação simples dada por uma proporcionalidade direta entre as duas variáveis.

(II) Clockwise (sentido horário) - é o tipo de curva mais comum de histerese e ocorre quando o pico de Css ocorre antes do pico de vazão.

(III) Counterclockwise (sentido anti-horário) - é a situação oposta do sentido horário, isto é, o pico de Css ocorre após o pico da vazão, formando uma curva com início e término em sentido anti-horário.

(IV) Single line plus - uma linha curva ou reta acrescida de uma volta, essa histerese ocorre raramente na natureza. Esta histerese é linear para uma determinada vazão que se torna uma relação circular para maiores taxas de vazão.

E (V) Figure eight - possui baixa frequência e ocorre em situações semelhantes as curvas horárias e anti-horárias.

A relação de Q e Css inicia-se com uma curva no sentido horário e termina com uma curva no sentido anti-horário, ou em ordem inversa, no sentido horário.

$\mathrm{Na}$ análise das histereses duas situações ocorrem, uma de termos o pico de Css na fase descendente da hidrógrafa, ou no seu pico, e outra na fase descendente da hidrógrafa.

Os laços de histerese podem ocorrer em diferentes direções, descrevendo as relações entre vazão e a concentração de sedimento nos diferentes eventos (Jasson, 2002).

As condições locais, como natureza geológica, tipo de uso da terra, relevo, tipo de solo e clima exercem significativo efeito no regime hidrossedimentológico (Hasnain \& Thayyen, 1999).

Enquanto, que as variáveis precipitação, vazão, concentração de sedimento em suspensão e descarga de sedimentos de arraste caracterizam os processos hidrossedimentológicos (Minella, 2007; Minella \& Merten, 2011).

A relação Q e Css é influenciada pela quantidade, intensidade e distribuição da chuva; variação temporal da taxa de escoamento superficial e forma do hidrograma; pelos tipos de uso e manejo do solo, que podem beneficiar ou dificultar processos erosivos; declividade da bacia hidrográfica; temperatura antecedente; condições de umidade e descarga; disponibilidade de sedimentos; distâncias percorridas pelos sedimentos (Williams, 1989; Reid et al., 1997; Seeger et al., 2004; Sammori et al., 2004); e conectividade hidrológica vertente canal fluvial.

Segundo Picouet et al. (2001) e Nondin Jr., (1985), as curvas de histerese são causadas, entre outras razões, pela variação da quantidade de material disponível para erosão, e diante desse conhecimento as curvas-chave são modelos empíricos, que representam não somente a capacidade do curso d'água de erodir as margens, o leito e de transportar os materiais das vertentes, mas também a disponibilidade de sedimentos na bacia, que tende a diminuir sua concentração durante a cheia.

Assim, estudar a dinâmica hidrossedimentológica, a partir dos tipos de histerese, possibilita compreender os mecanismos de transporte de sedimentos, inferir a localização das fontes de sedimentos suspensos e o grau de conectividade hidrossedimentológica.

Neste cenário, o presente trabalho teve como objetivo classificar e analisar as histereses da bacia do rio Guabiroba, localizada no centro-sul do estado do Paraná. 


\section{MATERIAIS E MÉTODOS}

\section{Área de Estudo}

A bacia do rio Guabiroba (BRG), área de estudo, localiza-se em Guarapuava, região centrosul do estado do Paraná, no Terceiro Planalto Paranaense e situa-se entre as coordenadas geográficas $25^{\circ} 23^{\prime}$ e $25^{\circ} 25^{\prime}$ de latitude Sul e 51 '20’ $51^{\circ} 25^{\prime}$ longitude W (Figura 1). A bacia possui área de $23 \mathrm{~km}^{2}$, densidade da drenagem de 3,18 $\left(\mathrm{km} / \mathrm{km}^{2}\right)$ e uma rede de drenagem de $4^{\circ}$ ordem, segundo a hierarquização fluvial de Strahler (1952, 1957; Thomaz, 2007).

As precipitações ao longo do ano apresentam médias mensais acima de $100 \mathrm{~mm}$; com variações extremas consideráveis, destaca-se o ano de 1985 (mínima) e 1983 (máxima), com 1.262mm e $3.168 \mathrm{~mm}$, respectivamente. A temperatura média anual varia de 16 a $17,5^{\circ} \mathrm{C}$, com média anual de $17^{\circ} \mathrm{C}$; os meses mais frios são junho e julho, enquanto janeiro e fevereiro são os mais quentes (Thomaz \& Vestena, 2003). O tipo de chuva predominante é a ciclônica ou frontal, ocasionada pela ação da massa polar atlântica (Thomaz \& Vestena, 2003).

Os principais tipos de solos são formados a partir do substrato rochoso basáltico de origem ígnea efusiva. Na BRG os tipos de solos são de textura argilosa, em vertentes com maiores declividades têm-se afloramento da rocha e solo com granulometria pedregosa, em especial os Litólicos Álicos (Neossolos Litólicos) e Cambissolos Álico (Embrapa, 2006).

A vegetação na bacia é de Floresta Ombrófila Mista, também conhecida como "Mata de Araucária”, registra-se também porções de campos abertos (IBGE, 2012).

Na BRG as matas estão presentes em 39,9\% da área, as formações arbóreas são resquícios da Floresta Ombrófila Mista, compostas por matas primárias e secundárias que se encontram na alta vertente e topos (Figura 2). As pastagens ocupam diferentes declividades, e recobrem a segunda maior porção da bacia com $31,7 \%$. Por ser uma área de difícil mecanização a criação de animais se mostra uma prática eficiente, com retorno financeiro e não demanda muitos recursos no manejo do solo. A agricultura mecanizada, conta com 6,07\% de área, e mesmo sendo uma porção pequena, essa atividade ocupa uma área significativa do terraço fluvial, merecendo atenção, em razão de conectividade hidrológica com o canal fluvial (Thomaz, 2007). A capoeira e a agricultura tradicional, representam 19,1\% do total da área da bacia, e as capoeiras são frequentemente utilizadas pelos agricultores no período de plantio.

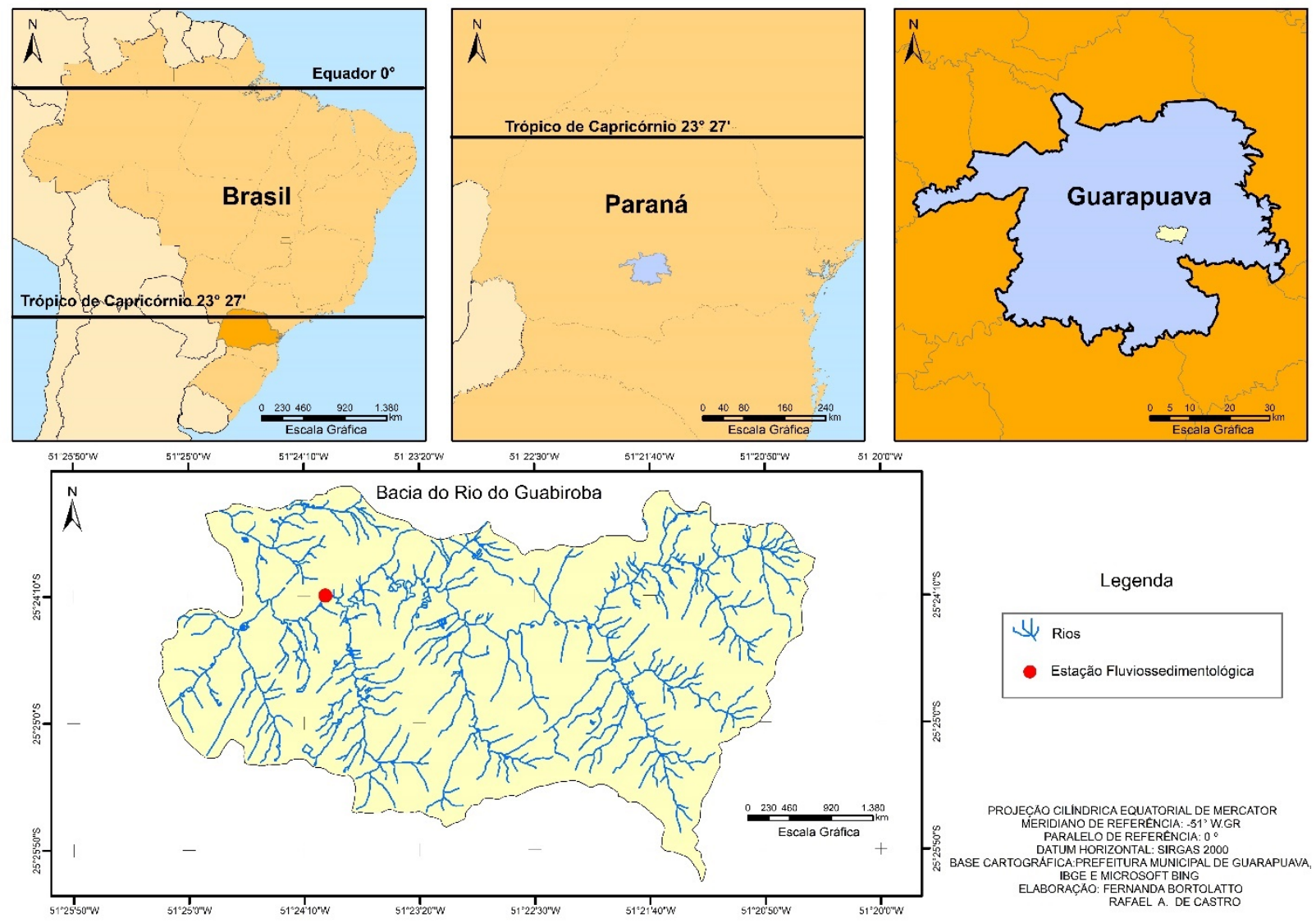

Figura 1 - Localização da Bacia do Rio Guabiroba, no município de Guarapuava, Paraná e Brasil. 


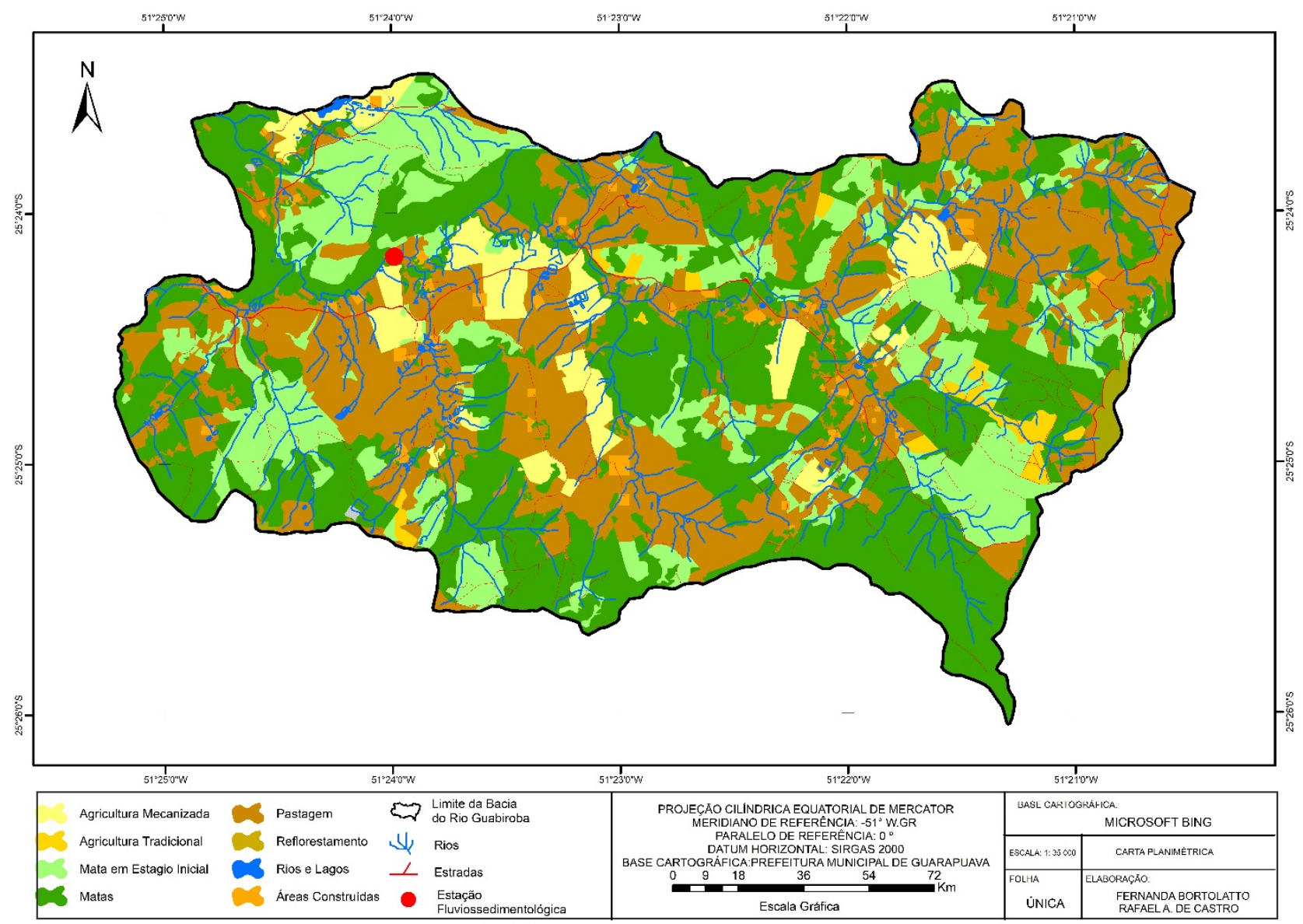

Figura 2 - Uso da terra na Bacia do Rio Guabiroba.

Na BRG o sistema agrícola mais utilizado é o tradicional, nesse sistema, começa-se a preparar a terra em junho e em setembro (final do inverno) inicia-se a queimada da capoeira; e posteriormente realiza-se o plantio principalmente das culturas do milho e feijão.

Nos meses de novembro a dezembro são realizados os tratos culturais (capina e herbicida), em janeiro inicia-se a colheita do feijão e em março a colheita do milho, após a colheita o solo inicia um período de regeneração nos meses de junho a agosto, para um novo ciclo agrícola (Thomaz, 2007).

As fontes de sedimento mais caracterizadas na BRG são as estradas rurais e os carreadores utilizados, essencialmente, na retirada de madeira (Thomaz et al., 2011). As estradas, carreadores e caminhos cruzam ou terminam às margens dos rios aumentando a entrada de sedimento no canal fluvial (Thomaz, 2007; Cunha, 2010).

\section{Dados e Procedimentos Utilizados}

Os dados de vazão, concentração de sedimento em suspensão e pluviosidade foram monitorados automaticamente por uma estação fluviossedimentológica e pluviógrafo, em intervalo temporal de quinze minutos, situada na latitude $25^{\circ} 24^{\prime} 19^{\prime \prime}$ S e longitude $51^{\circ} 24^{\prime} 10^{\prime}$ ' W (Figura 1).
Para o estudo, selecionou-se vinte eventos hidrológicos representativos de condições hidrológicas seguindo o critério de precipitação superior a $8 \mathrm{~mm}$, precipitação no dia anterior ao evento e sem ocorrência e com variabilidade no tempo de duração do evento (TDE). O TDE foi obtido a partir da soma do tempo de duração do evento, desde o início de alteração no dado de Css, no início do ramo acrescente do sedimentograma e, até o último dado do ramo decrescente da curva de Css.

Na sequência, obteve-se de cada evento hidrológico, os seguintes gráficos: (1) hidrograma (Q x tempo); (2) sedimentograma (Css x tempo); (3) hietograma (Chuva $x$ tempo); e (4) histerese (Css x Q).

Em seguida, classificou-se as histereses de acordo com os modelos (Figura 3) descritos por Williams (1989) e analisou-se qualitativamente as histereses em função das características físicas da bacia e das condições hidrológicas ocorridas no evento.

Posteriormente, fez-se uma análise de regressão e correlação entre a vazão e Css e obteve-se o coeficiente de determinação $\left(\mathrm{r}^{2}\right)$ e a equação da reta de regressão linear $(\mathrm{Y}=\mathrm{b}+\mathrm{aX})$ para o evento todo na ascensão e recessão do hidrograma. 

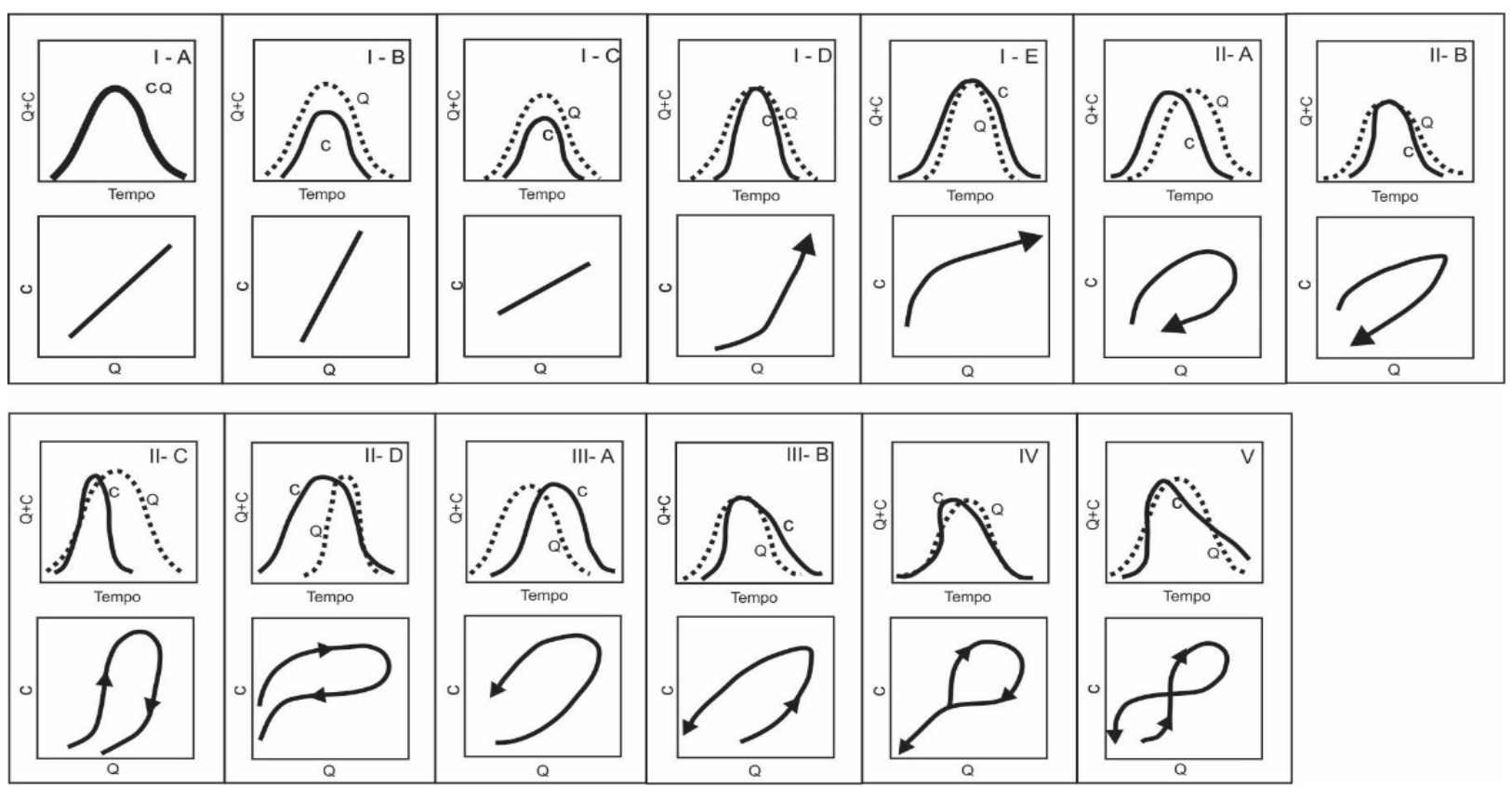

Figura 3 - Modelos de relação entre Css e vazão representados pela curva de histerese.

Notas: (1) C é a concentração de sedimentos, e Q é a vazão ou descarga fluvial. (2) Classe I - única linha (reta ou curva) (singlevalued line): (Ia) linha reta de $45^{\circ}$; (Ib) linha reta com inclinação $>1$; (Ic) linha reta com inclinação < 1; (Id) ângulo voltandose para cima; (Ie) ângulo voltando-se para baixo. Classe II - curva no sentido horário (clockwise loop): (IIa) concentração chegando ao pico antes da vazão; (IIb) picos simultâneos de água e sedimento; (IIc) curvas assimétricas - expansão da concentração menor que a vazão, com a concentração chegando ao pico primeiro; (IId) curvas assimétricas - expansão da concentração e maior que a vazão, com a concentração chegando ao pico primeiro. Classe III - curva no sentido anti-horário (counterclockwise loop): (IIIa) vazão chegando ao pico antes da concentração de sedimento; (IIIb) picos de vazão e concentração são simultâneos. Classe IV - linha única acrescida de uma volta (single line plus loop). Classe V - figura oito (figure eight). Fonte: Williams (1989).

\section{RESULTADO E DISCUSSÃO}

Os eventos mostraram-se representativos das condições hidrológicas atuantes na bacia hidrográfica. A tabela 1, sintetiza os dados de precipitação, vazão e Css de cada um dos eventos. A precipitação total nos eventos variou de $9,6 \mathrm{~mm}$ a $62,0 \mathrm{~mm}$ e a intensidade máxima de pluviosidade oscilou de 2,0 a $24,4 \mathrm{~mm}$ em intervalo temporal de quinze minutos. A Css máxima registrada foi de $886 \mathrm{mg} / \mathrm{L}$ e a mínima de $23,9 \mathrm{mg} / \mathrm{L}$. A vazão variou de $0,93 \mathrm{~m}^{3} / \mathrm{s}$ a $41,64 \mathrm{~m}^{3} / \mathrm{s}$. De modo geral, contatou-se que os eventos que apresentaram maiores volumes de precipitação, também foram os que tiveram maiores vazões e Css máxima.

Em síntese, observou-se que os eventos que apresentaram chuvas mais intensas foram também os que constatou-se os maiores valores de Css comprovado por um coeficiente de determinação $\left(\mathrm{r}^{2}\right)$ de 0,77 . Enquanto, a relação entre o volume e a Css máxima demonstrou uma correlação positiva pequena, dada por um $\mathrm{r}^{2}$ de 0,23 . Apesar da duração, intensidade e volume das chuvas influenciarem no escoamento superficial, remoção das partículas, transporte, deposição dos sedimentos e onda de vazão, não se observou um padrão na relação Css e vazão específica, devido as características da precipitação. Para Seeger et al. (2004), a maioria das histereses com sentido antihorário podem ser explicadas pelo maior volume precipitado e/ou pela condição de umidade antecedente do solo ao evento.

$\mathrm{Na}$ tabela 2, têm-se o coeficiente de determinação e a equação de regressão linear dos dados de Css e vazão total do evento, da ascensão e regressão do hidrograma. A correlação entre Css e vazão total nos eventos indicaram $\mathrm{r}^{2}$ superiores a 0,7 em seis dos eventos e superiores a 0,5 em dezesseis dos eventos. Enquanto, no evento 12 registrou-se um $\mathrm{r}^{2}$ praticamente nulo entre a Q e a Css, esse resultado demostra que a dinâmica fluviossedimentológica pode ser condicionada por outros fatores independentes da vazão, a exemplo da ação direta de animais no curso fluvial - que possibilitam a disponibilização de grandes quantidades de sedimentos finos para o transporte. Os menores índices de correlação foram encontrados nas histereses do tipo II - clockwise loop e V - figure eight. Os índices de correlação entre Css e vazão na recessão do hidrograma mostraram-se melhores do que na ascensão, devido a carga de lavagem, a qual impacta significativamente na Css. 
Tabela 1 - Síntese de dados hidrossedimentológicos dos eventos selecionados, na bacia do Rio Guabiroba.

\begin{tabular}{|c|c|c|c|c|c|c|c|}
\hline Evento & $\begin{array}{c}\text { Data/Hora } \\
\text { de início }\end{array}$ & $\begin{array}{l}\text { Data/Hora } \\
\text { de término }\end{array}$ & TDE & $\begin{array}{c}\text { P (mm) } \\
\text { Total }\end{array}$ & $\begin{array}{c}\text { P (mm) } \\
\text { Máx. 15min }\end{array}$ & $\begin{array}{l}\text { Q (m³/s) } \\
\text { Máx. }\end{array}$ & $\begin{array}{l}\text { Css (mg/L) } \\
\text { Máx. }\end{array}$ \\
\hline 1 & $17 / 01 / 09-07: 00$ & $18 / 01 / 09-13: 00$ & $30 \mathrm{~h}$ & 16,6 & 6,6 & 1,84 & 107,6 \\
\hline 2 & $27 / 02 / 09-06: 15$ & 28/02/09-07:15 & $24 \mathrm{~h}$ & 22,4 & 4,8 & 1,77 & 63,9 \\
\hline 3 & $13 / 05 / 09-03: 45$ & $16 / 05 / 09-10: 15$ & $79 \mathrm{~h}$ & 41,8 & 2,6 & 1,91 & 84,4 \\
\hline 4 & $12 / 10 / 09-00: 00$ & $12 / 10 / 09-22: 45$ & $10 \mathrm{~h}$ & 42,6 & 7,6 & 3,79 & 119,8 \\
\hline 5 & $14 / 10 / 09-17: 30$ & 15/10/09-08:15 & $16 \mathrm{~h}$ & 40,4 & 6,0 & 2,69 & 92,8 \\
\hline 6 & $15 / 10 / 09-21: 15$ & $16 / 10 / 09-21: 45$ & $24 \mathrm{~h}$ & 18,9 & 2,1 & 2,36 & 58,6 \\
\hline 7 & $18 / 10 / 09-16: 30$ & $19 / 10 / 09-06: 45$ & $14 \mathrm{~h}$ & 15,1 & 3,2 & 2,63 & 122,8 \\
\hline 8 & $19 / 10 / 09-05: 45$ & $19 / 10 / 09-22: 30$ & $16 \mathrm{~h}$ & 10,2 & 2,0 & 2,27 & 49,3 \\
\hline 9 & $20 / 10 / 09-10: 15$ & $21 / 10 / 09-23: 45$ & $37 \mathrm{~h}$ & 49,6 & 21,4 & 2,58 & 321,9 \\
\hline 10 & $11 / 12 / 09-23: 45$ & $12 / 12 / 09-23: 45$ & $24 \mathrm{~h}$ & 69,0 & 8,8 & 7,10 & 234,8 \\
\hline 11 & $16 / 12 / 09-15: 45$ & $17 / 12 / 09-11: 00$ & $19 \mathrm{~h}$ & 20,2 & 6,2 & 1,79 & 67,09 \\
\hline 12 & $17 / 12 / 09-08: 45$ & 18/12/09-10:15 & $25 \mathrm{~h}$ & 43,4 & 24,4 & 41,64 & 886,0 \\
\hline 13 & $24 / 12 / 09-11: 45$ & 25/12/09-10:15 & $23 \mathrm{~h}$ & 26,6 & 8,8 & 2,59 & 141,4 \\
\hline 14 & $03 / 01 / 10-13: 45$ & $04 / 01 / 10-12: 15$ & $23 \mathrm{~h}$ & 9,9 & 3,2 & 1,47 & 38,6 \\
\hline 15 & $10 / 01 / 10-10: 30$ & $11 / 01 / 10-11: 15$ & $25 \mathrm{~h}$ & 27,2 & 5,4 & 1,17 & 28,78 \\
\hline 16 & $11 / 01 / 10-12: 15$ & $12 / 01 / 10-14: 30$ & $26 \mathrm{~h}$ & 21,4 & 6,4 & 2,25 & 130,5 \\
\hline 17 & $13 / 01 / 10-02: 15$ & $13 / 01 / 10-13: 30$ & $12 \mathrm{~h}$ & 23,6 & 4,6 & 1,13 & 172,0 \\
\hline 18 & $13 / 01 / 10-14: 30$ & $14 / 01 / 10-01: 45$ & $11 \mathrm{~h}$ & 13,8 & 3,2 & 4,77 & 79,5 \\
\hline 19 & 20/01/10 - 03:15 & $21 / 01 / 10-09: 15$ & $30 \mathrm{~h}$ & 35,8 & 7,0 & 3,53 & 173,3 \\
\hline \multirow[t]{4}{*}{20} & $22 / 01 / 10-02: 45$ & 23/01/10 - 06:15 & $27 \mathrm{~h}$ & 9,6 & 2,8 & 0,93 & 23,9 \\
\hline & & Mínima & & 9,6 & 2,0 & 0,93 & 23,9 \\
\hline & & Máxima & & 69,0 & 24,4 & 41,64 & 886,0 \\
\hline & & Média & & 26,2 & 6,9 & 4,3 & 154,0 \\
\hline
\end{tabular}

*Nota: P é a precipitação, Q a vazão, Css a Concentração de sedimento em suspensão, e TDE o tempo de duração do evento em horas.

Tabela 2 - Coeficiente de determinação e equação de regressão linear da Css (mg/L) versus a vazão (m³/s) total, na ascensão e recessão do hidrograma, para os eventos monitorados na bacia hidrográfica do Rio Guabiroba.

\begin{tabular}{|c|c|c|c|c|c|c|c|}
\hline \multicolumn{4}{|c|}{ Q/Css - Total } & \multicolumn{2}{|l|}{ Q/Css - Ascenção } & \multicolumn{2}{|l|}{ Q/Css - Recessão } \\
\hline Classe* & $\mathbf{E}$ & Equação & $\mathbf{r}^{2}$ & Equação & $\mathbf{r}^{2}$ & Equação & $\mathbf{r}^{2}$ \\
\hline I & 6 & $y=0,2867 x+1,753$ & 0,9942 & $y=0,2883 x+1,746$ & 0,9974 & $y=0,2596 x+1,8029$ & 0,9986 \\
\hline \multirow{7}{*}{ II } & 4 & $y=27,466 x-17,037$ & 0,6052 & $y=23,029 x+22,758$ & 0,6252 & $y=110,07 x-26,551$ & 0,9255 \\
\hline & 5 & $\mathrm{y}=13,251 \mathrm{x}+8,2365$ & 0,5443 & $y=17,833 x+7,4927$ & 0,8931 & $y=14,498 x-9,1218$ & 0,8931 \\
\hline & 10 & $y=15,776 x+0,6387$ & 0,4539 & $y=58,464 x-19,081$ & 0,9660 & $y=17,1 x-9,6168$ & 0,4330 \\
\hline & 12 & $y=0,2816 x+174,96$ & $\mathbf{0 , 0 0 0 3}$ & $y=93,191 x-78,452$ & 0,6994 & $y=0,9907 x+142,79$ & 0,0049 \\
\hline & 13 & $y=32,065 x-9,2872$ & 0,7110 & $y=48,309 x-16,361$ & 0,7665 & $y=25,719 x-5,0771$ & 0,9404 \\
\hline & 14 & $y=30,731 x-11437$ & $\mathbf{0 , 8 1 1 7}$ & $y=47,621 x-20,343$ & 0,7921 & $y=34,071 x-16,456$ & 0,9525 \\
\hline & 19 & $y=39,593 x-30,875$ & 0,6019 & $\mathrm{y}=47,07 \mathrm{x}-10,854$ & 0,6975 & $y=30,633 x-26,609$ & 0,9778 \\
\hline \multirow{2}{*}{ III } & 11 & $y=110,61 x-189,51$ & 0,5724 & $y=92,008 x-160,22$ & 0,6517 & $y=234,75 x-423,46$ & 0,9760 \\
\hline & 20 & $y=83,528 x-64,789$ & 0,5294 & $y=5,7572 x-0,5357$ & 0,0297 & $y=85,987 x-66,502$ & 0,5375 \\
\hline \multirow{2}{*}{ IV } & 9 & $y=98,081 x-82,097$ & 0,5023 & $y=135,6 x-143,12$ & 0,8606 & $y=1083,3 x-2574,3$ & 0,6207 \\
\hline & 18 & $y=19,197 x-19,171$ & 0,9105 & $y=19,599 x-19,957$ & $\mathbf{0 , 8 9 1 7}$ & $y=36,21 x-9816$ & 0,8774 \\
\hline \multirow{11}{*}{ V } & 1 & $y=94,752 x-18,367$ & 0,2214 & $y=353,01 x+120,48$ & 0,4696 & $y=72,784 x-11,628$ & 0,7897 \\
\hline & 2 & $y=67,784 x-6,1086$ & 0,2272 & $\mathrm{y}=112,97 \mathrm{x}-17,936$ & 0,3921 & $\mathrm{y}=81,950 \mathrm{x}-17,400$ & 0,9188 \\
\hline & 3 & $y=107,71 x+24,366$ & 0,7162 & $y=46,373 x+21,946$ & 0,0772 & $y=0,0078 x+0,288$ & 0,9288 \\
\hline & 7 & $y=0,0233 x+1,1522$ & 0,7719 & $y=0,0193 x+1,105$ & $\mathbf{0 , 8 8 4 7}$ & $y=0,0241 x+1,3444$ & 0,9497 \\
\hline & 8 & $y=157,24 x-169,45$ & 0,6351 & $y=219,82 x-241,3$ & 0,7366 & $y=162,62 x-176,08$ & 0,5896 \\
\hline & 15 & $y=17,744 x+4,5338$ & 0,6061 & $y=13,893 x+9,3905$ & 0,4623 & $y=19,551 x+2,1615$ & 0,8019 \\
\hline & 16 & $y=65,14 x-37,205$ & 0,5303 & $y=42,608 x-26,882$ & 0,4228 & $y=76,97 x-45,906$ & 0,7722 \\
\hline & 17 & $y=1178,1 x-1224$ & 0,6391 & $y=884,68 x-862,31$ & 0,7224 & $y=1003 x-1027$ & 0,2673 \\
\hline & & Mínima & 0,0003 & & 0,0297 & & 0,2673 \\
\hline & & Máxima & 0,9942 & & 0,9974 & & 0,9986 \\
\hline & & Média & 0,6196 & & 0,6195 & & 0,7671 \\
\hline
\end{tabular}

Nota: * Classe de histerese, conforme a classificação de Williams (1989) (Figura 3). E é o número do evento (Tabela 1). Na equação o y representa a vazão (m³/s), e o $x$ a Css (mg/L). 
Das vinte histereses dos eventos selecionados, uma apresentou figura do tipo single-valued line (única linha reta ou curva), sete clockwise loop (curva no sentido horário), duas conterclokwise loop (curva no sentido anti-horário), duas single line plus loop (linha única acrescida de uma volta), e oito figure eight (figura oito) (Tabela 2). Observou-se que a dinâmica hidrossedimentológica na bacia hidrográfica não apresentou um comportamento único, dado pelas diversidades de tipo de histerese, em todos os modelos descritos por Williams (1989), colaborando com os resultados dos estudos em bacias hidrográficas rurais que indicam haver mais de um tipo de histerese (Lenzi \& Marchi, 2000; Seeger et al, 2004; Vestena, 2009; Minella et al., 2011).

Na figura 4, têm-se histereses representativas dos modelos da relação Css e vazão mais comuns na BRG, do tipo II - clockwise loop e V - figure eight. Nos eventos 4, 5, 10, 12, 13, 14 e 19 tevese histerese clockwise loop, caracterizada por apresentar pico de Css antecedendo o pico da vazão, assim como por um decréscimo da Css anterior ao pico de vazão, ou seja, apresentando

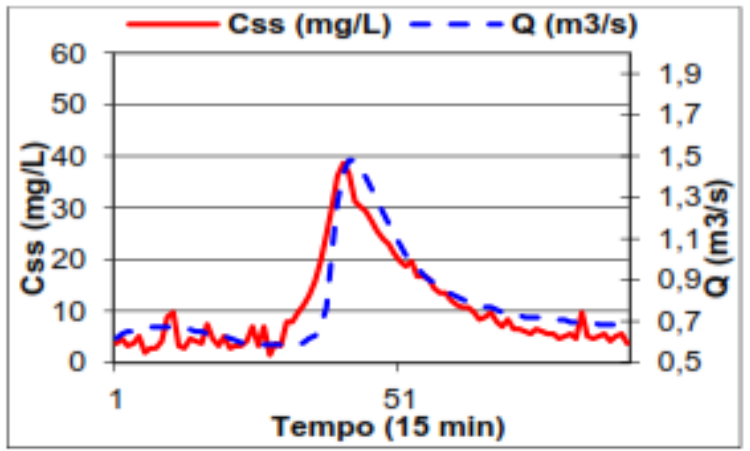

(a)

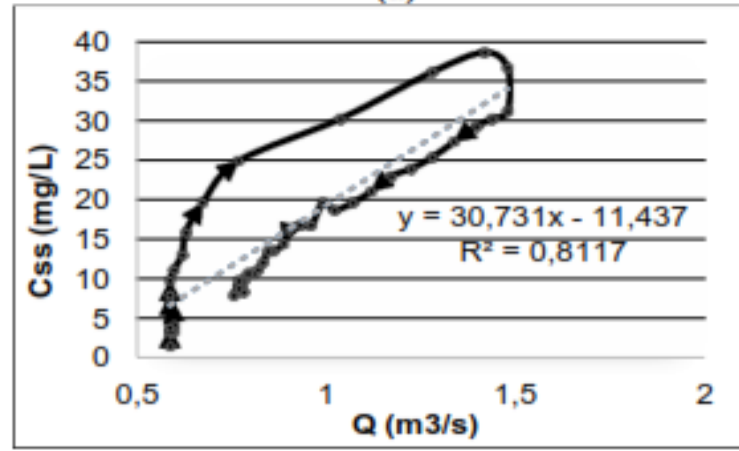

(b)

II - Histerese clockwise loop é uma curva dada em função da ocorrência do pico de Css antes do pico de vazão, e por um decréscimo na Css antes da vazão atingir seu pico de ascensão. um decréscimo da Css ainda durante a ascensão do hidrograma. Enquanto, nos eventos 1, 2, 3, 7, 8, 15, 16 e 17 histerese do tipo figure eight, condicionado principalmente por apresentar mais de um pico de Css. A presença de mais de um pico de Css dar-se em função de sedimentos provenientes de diferentes fontes, em que o primeiro pico de Css é decorre de sedimentos oriundos de fontes próximas ao canal de drenagem e os demais picos de outras fontes distantes (Lopes et al., 2007; Vestena, 2009), ambos em função do uso e manejo do solo. Cabe ressaltar que o primeiro pico de Css, logo após o início da chuva, antes do pico da vazão é determinado, essencialmente, pela carga de lavagem (Vestena, 2009). Constatou-se ainda haver nos eventos 3, 7, 15 e 16 um declive do ramo descendente da vazão mais acentuado do que o ramo da Css. Essa característica pode indicar que após a ocorrência do pico de Css, a disponibilidade e o transporte de sedimentos são suficientemente elevados, fazendo com que a Css decresça lentamente com o tempo, enquanto a $\mathrm{Q}$ decresce mais rapidamente como destacado por Lefrançois et al. (2007).

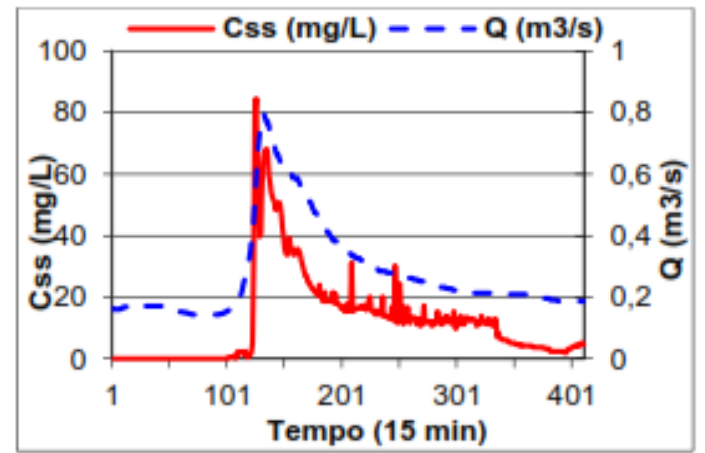

(a)

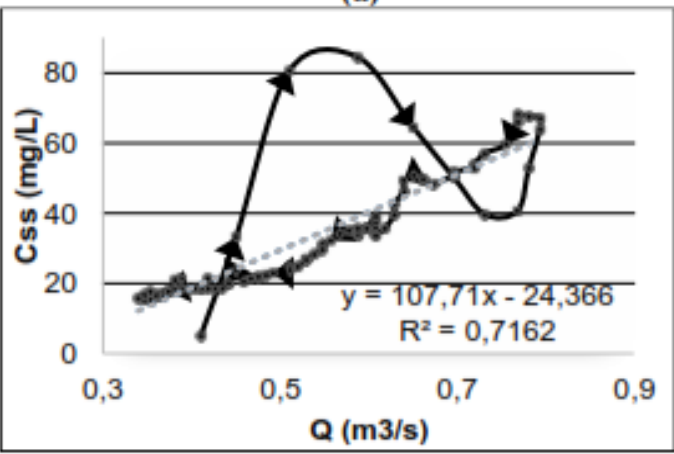

(b)

V - Histerese figure eight é uma curva com início no sentido clockwise terminada no sentido counterclowise ou vice-versa, decorrente da ocorrência de dois picos de Css, enquanto, tem-se apenas um pico de vazão.

Figura 4 - Hidrograma e sedimentograma (a) e histerese (b): (1) Evento 14 e (2) Evento 3. 
A partir da dinâmica hidrossedimentológica encontrada na BRG, verificou-se que os sedimentos oriundos das áreas agrícolas demoram maior tempo para chegarem aos cursos fluviais, diferentemente dos sedimentos provenientes das estradas rurais não pavimentadas, após o início da chuva, que rapidamente são carreados aos cursos fluviais. Uma vez, que as estradas rurais não pavimentadas sobrepostas a canais fluviais ocasionam incremento na concentração de sedimentos em suspensão na BRG (Thomaz et al. 2014).

Os eventos que apresentaram histereses clockwise loop e figure eight ocorreram em época de plantio, trato das culturas e colheita das principais culturas cultivadas na BRG. Os eventos 7 e 8 ocorreram na época de plantio das culturas de milho e feijão, o evento 1 no período de trato das culturas e início de colheita, e os eventos 2, 3, 15, 16 e 17, no período de colheita das respectivas culturas.

As atividades agrícolas condicionam a existência de sedimentos disponíveis para o transporte, em consonância com o resultado da pesquisa realizada em bacia hidrográfica no sul do Brasil por Minella et al., 2009, que avaliaram o aporte de sedimentos provenientes de lavouras contribuíram com 64\% dos sedimentos e as estradas com $36 \%$, assim como, que as fontes, são variáveis no tempo em função do manejo do solo e das obras realizadas nas estradas.

Os demais tipos de histerese também se fizeram presentes na BRG, a single-valued line, counterclockwise loop e single line plus loop (Figura 5). A histerese single - valued line deuse no evento 6 , onde teve-se praticamente uma sobreposição do hidrograma e sedimentograma (Figura 5(1)) dado por um $\mathrm{r}^{2}$ de 0,99, o maior índice obtido dentre os 20 eventos. Essa relação linear indica que a $\mathrm{Q}$ e a Css apresentaram resposta hidrossedimentológica sincronizada, demonstrando picos simultâneos. Já, histerese counterclockwise loop, nos eventos 11 e 20, têmse pico de vazão antes do pico de Css, expondo um retardo na resposta sedimentológica, decorrente de carga de sedimentos provenientes de áreas mais distantes. A histerese single line plus loop, nos eventos 9 e 18 está relacionada aos três tipos anteriores, e por essa razão suas determinantes possuem as mesmas caracte-rísticas. Segundo Baca (2010), a simultaneidade da relação vazão Css na ascensão do hidrograma permite inferir que as fontes de sedimentos estavam próximas ou, provindas de algum banco de sedimentos dentro do canal de drenagem.

e

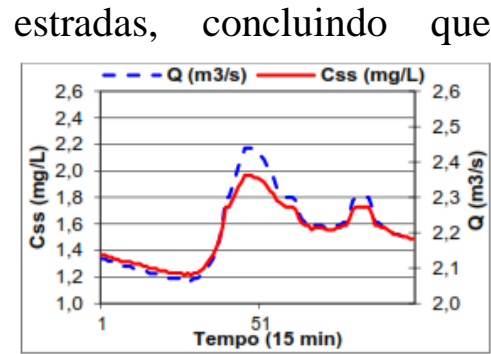

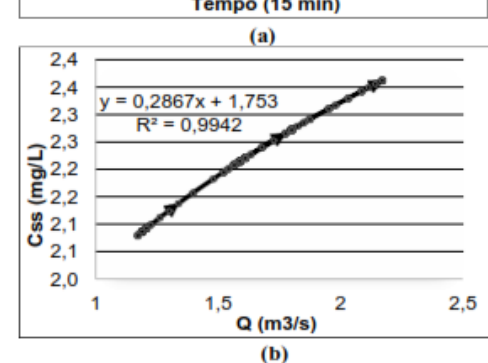

I - Histerese single - valued line presença de uma resposta da vazão sincronizada com a Css.

(1)

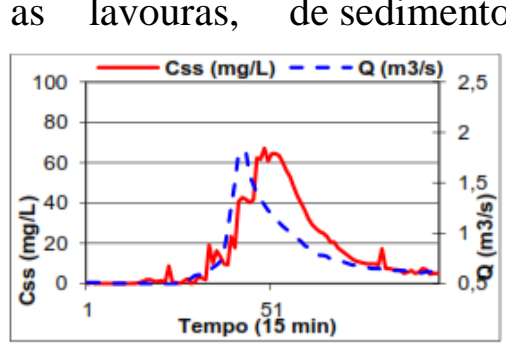

(a)

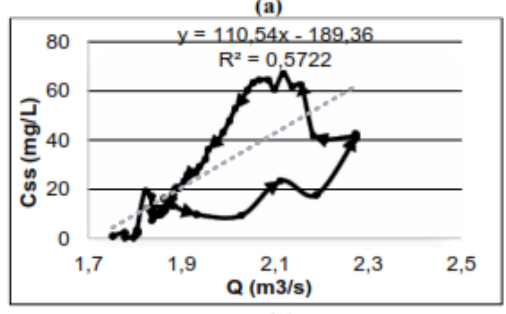

(b)

III - Histerese counterclockwise loop é uma curva dada em função da vazão apresentar pico antes da Css.
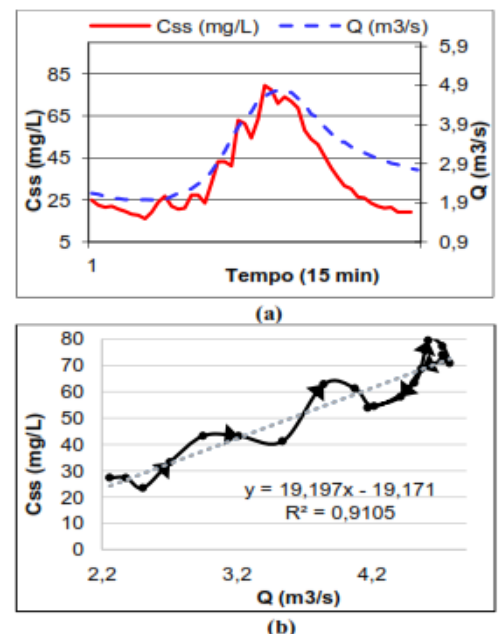

IV - Histerese single line plus loop é a junção das histereses single valued line e clockwise loop ou counterclockwise loop.

(3)

Figura 5 - Hidrograma e sedimentograma (a) e histerese (b): (1) Evento 6, (2) Evento 11 e (3) Evento 18.

\section{CONCLUSÕES}

Na BRG não se verificou um tipo único de histerese entre a $\mathrm{Q}$ e Css. A diversidade de modelos de histerese obtidos na BHG, aponta para uma complexidade de condições locais atuantes na carga fluvial de sedimentos finos transportada. As histereses clockwise loop e figure eight ocorreram em $75 \%$ dos eventos analisados, indicando que as áreas de 
contribuição de sedimentos finos na BRG decorrem de fontes diversas, inicialmente de estradas rurais próximas ou sobrepostas aos cursos fluviais, e posteriormente, de áreas agrícolas, em função do manejo do solo, principalmente no cultivo do milho, soja e feijão.
A compreensão e classificação da histerese, possibilitou compreender a dinâmica de produção e transporte de sedimentos em suspensão e inferir sobre processos erosivos atuantes na bacia hidrográfica que deterioram a qualidade do solo e da água.

\section{AGRADECIMENTOS}

Os autores agradecem o CNPq e o Grupo de Pesquisa Hidrogeomorfologia pelo apoio e as sugestões do Prof. Dr. Edivaldo Lopez Thomaz. Este artigo faz parte da dissertação de mestrado da autora principal.

\section{REFERÊNCIAS}

BACA, P. Hysteresis effect in suspended sediment concentration in the Rybárik basin, Slovakia. Hydrological Sciences Journal, v. 53, n. 1, p. 224-235, 2008.

CUNHA, M.C. Caracterização das estradas rurais não pavimentadas como elementos presentes na paisagem da bacia hidrográfica do rio das Pedras, Guarapuava-PR. Rev. GEOMAE, v. 1, n.2, p. 73 - 91, 2010.

EMBRAPA. Centro Nacional de Pesquisa de Solos. Sistema brasileiro de classificação de solos. 2. ed. Rio de Janeiro, 2006.

HASNAIN, S.I. \& THAYYEN, R. J. Discharge and suspendedsediment concentration of meltwaters, draining from the Dokriani glacier, Garhwal Himalaya, India. Journal of Hydrology, v. 218, p. 191-198, 1999.

IBGE. Instituto Brasileiro de Geografia e Estatística. Manual Técnico da Vegetação Brasileira. 2. ed. Rev. e Amp. Rio de Janeiro, 271 p., 2012.

JANSSON, M.B. Determining sediment sources areas in a tropical river basin, Costa Rica. Catena, v. 47, p.63-84, 2002.

KLAR, A.E. A água no sistema solo-planta-atmosfera. São Paulo: Nobel, 488 p., 1984.

KLEIN, M. Anticlockwise hysteresis in suspended sediment concentration during individual storms. Catena, v. 11, p. 251-257, 1984.

LEFRANÇOIS, J.; GRIMALDI, C.; GASCUEL-ODOUX, C.; GILLIET, N. Suspended sediment and discharge relationships to identify bank degradation as a main sediment source on small agricultural catchments. Hydrological Processes, v. 21, n. 15, p. 2923-2933, 2007.

LENZI, M.A. \& MARCHI, L. Suspended sediment load during floods in a small stream of the Dolomites. Catena, v. 39, p. 267 282, 2000.

LOPES, N.H.Y. KOBIYAMA, M.; SANTOS, I. Relação entre concentração de sedimentos em suspensão e vazão em microbacias experimentais na região norte do estado de Santa Catarina, Brasil. In: SIMPÓSIO BRASILEIRO DE RECURSOS HÍDRICOS, 17, 2007, São Paulo. Anais... São Paulo: ABRH, 2007.12 p.

MINELLA, J.P. MERTEN, G.H. CLARKE, R.T. Método "fingerprinting" para identificação de fontes de sedimentos em bacia hidrográfica rural. Rev. Bras. Eng. Agri. Amb., v. 13, n. 5, p. 633-638, 2009.

MINELLA, J.P.G. \& MERTEN, G.H. Monitoramento de bacias hidrográficas para identificar fontes de sedimentos em suspensão. Ciência Rural, v. 41, n. 3, p. 424-432, 2011.

MINELLA, J.P.G., MERTEN, G. H., REICHERT, J. M.; SANTOS, D. R. Identificação e implicações para a conservação do solo das fontes de sedimentos em bacias hidrográficas. Rev. Bras. Ciênc. Solo, v. 31, n. 6, p.1637-1646, 2007.

MINELLA, J.P.G.; MERTEN, G.H.; MAGNAGO, P. Análise qualitativa e quantitativa da histerese entre vazão e concentração de sedimentos durante eventos hidrológicos. Rev. Bras. Eng. Agri. Amb., v. 15, n. 12, p. 1306-1313, 2011.
PICOUET, C.; HINGRAY, B.; OLIVRY, J.C. Empirical and conceptual modeling of the suspended sediment dynamics in a large tropical African river: the Upper Niger river basin. Journal of Hydrology, v. 250, n. 1-2, p. 19-39, 2001.

REID, I.; BATHURST, J.C.; CARLING, P.A.; WALLING, D.E.; WEBB, B.W. Sediment Erosion, Transport and Deposition. In: THORNE, C.R.; HEY, R.D.; NEWSON, M.D. Applied Fluvial Geomorphology for River Engineering and Management. New York: John Wiley \& Sons, 1997. p. 95-135. SAMMORI, T.; YUSOP, Z.; KASRAN, B.; NOGUCHI, S.; TANI, M. Suspended solids discharge from a small forested basin in the humid tropics. Hydrological Processes, v. 18, p. 721-738, 2004.

SEEGER, M.; ERREA, M.P.; BEGUERÍA, S.; ARNÁEZ, J.; MARTÍ, C; GARCÍA-RUIZ, J. M. Catchment soil moisture and rainfall characteristics as determinant factors for discharge/suspended sediment hysteretic loops in a small headwater catchment in the Spanish pyrenees. Journal of Hydrology, v. 288, n. 3-4, p. 299-311, 2004

STOTT, T. Impacts of constructing a rural cycle way on suspended sediment transport processes. Catena, v. 68, p.16 - 24, 2006.

STRAHLER, A.N. Hypsometric (area-altitude) analysis of erosional topology. Geological Society of America Bulletin, v. 63, n. 11, p. 1117-1142, 1952.

STRAHLER, A.N. Quantitative analysis of watershed geomorphology. Transactions of the American Geophysical Union, v. 38, n. 6, p. 913-920, 1957.

THOMAZ, E. L.; ANTONELI, V.; DIAS, W.A. Estimativa de Proveniência de Sedimento em Cabeceira de Drenagem com Alta Densidade de Estradas Rurais não Pavimentadas. Revista Brasileira de Recursos Hídricos, v. 16, n. 2, p. 25-37, 2011.

THOMAZ, E.L. \& VESTENA, L.R. Aspectos Climáticos de Guarapuava-PR. Guarapuava: UNICENTRO, 106 p., 2003.

THOMAZ, E.L. Dinâmica do uso da terra e degradação do solo na bacia do Rio Guabiroba - Guarapuava/PR. R. RA'E GA, n. 13, p. 109-128, 2007.

THOMAZ, E.L.; VESTENA, L.R.; RAMOS-SCHARRÓN, C.E. The effects of unpaved roads on suspended sediment concentration of third- to fifth-order streams - A case study from southern Brazil. Water and Environment Journal, v. 28, p. 547-555, 2014.

VESTENA, L.R. Análise da dinâmica hidrossedimentológica em uma bacia hidrográfica no sul do Brasil. Sociedade \& Natureza, v. 21, n. 3, p. 413-424, 2009.

WILLIAMS, G.P. Sediment concentration versus water discharge during single hydrologic events in rivers. Journal of Hydrology. Amsterdam, v. 111, p. 89-106, 1989.

Submetido em 7 de junho de 2020 Aceito para publicação em 15 de junho de 2020 